\title{
Balloon Deflection Technique to Facilitate Stent Delivery in Impassable Situations During Percutaneous Coronary Intervention
}

\author{
Prakash Kumar ${ }^{\mathrm{a}}$, Santosh Kumar Sinha ${ }^{\mathrm{b}, \mathrm{c}}$, Najeeb Ullah Sofi ${ }^{\mathrm{b}}$, Mahmodullah Razi ${ }^{\mathrm{b}}$, \\ Awdesh Kumar Sharma ${ }^{\mathrm{b}}$, Umeshwar Pandey ${ }^{\mathrm{b}}$, Praveen Shukla ${ }^{\mathrm{b}}$, Ramesh Thakur ${ }^{\mathrm{b}}$, \\ Chandra Mohan Varma ${ }^{b}$, Vinay Krishna ${ }^{b}$
}

\begin{abstract}
Background: Long and multiple lesions especially in the background of tortuous coronary artery often require multiple stents. The entry and passage of another stent through an already deployed stent becomes challenging because of poorly expanded stent struts, calcified tissue, underlying tortuosity, highly angulated takeoff of the vessel, and lack of coaxiality. It can be facilitated by balloon deflection technique (BDT) where balloon at proximal edge of main vessel stent over a buddy wire helps to deflect second stent either distally or into side branch by blocking potential dead.

Methods: The present retrospective, single-center study included 16,189 consecutive patients who underwent percutaneous coronary intervention (PCI) either through transfemoral or transradial route from January 2014 to August December 2019 at LPS Institute of Cardiology, GSVM Medical College, Kanpur, UP, India where BDT was used in situation of impassable stent among 37 patients.
\end{abstract}

Results: The mean age of patients was $75.4 \pm 6.5$ years. The commonest clinical presentation was chronic coronary syndrome $(\mathrm{n}=$ $19 ; 51.3 \%$ ) followed by non-ST-segment elevation myocardial infarction (NSTEMI) $(\mathrm{n}=9 ; 24.4 \%)$, ST-segment elevation myocardial infarction (STEMI) $(\mathrm{n}=5 ; 13.5 \%)$, and unstable angina (UA) $(\mathrm{n}=10 ; 10.8 \%)$. Type B2 lesion was commonest $(45.9 \%)$ followed by type $\mathrm{C}(35.2 \%)$ and $\mathrm{B} 1(18.9 \%)$. The commonest indication for BDT was angulation $(\mathrm{n}=10 ; 27.1 \%)$ followed by tortuosity $(\mathrm{n}=$ 9; $24.3 \%)$, chronic total occlusion $(\mathrm{n}=8 ; 17.9 \%)$, calcification $(\mathrm{n}$ $=7 ; 18.9 \%)$, and distally located lesion $(\mathrm{n}=3 ; 8.1 \%)$. The left cir-

Manuscript submitted February 11, 2020, accepted June 15, 2020

Published online May 14, 2020

aDepartment of Cardiology, Rajendra Institute of Medical Science, Ranchi, Jharkhand, India

bepartment of Cardiology, LPS Institute of Cardiology, G.S.V.M. Medical College, Kanpur, Uttar Pradesh 208002, India

${ }^{c}$ Corresponding Author: Santosh Kumar Sinha, Department of Cardiology, LPS Institute of Cardiology, G.S.V.M. Medical College, Kanpur, Uttar Pradesh 208002, India. Email: fionasan@rediffmail.com

doi: https://doi.org/10.14740/cr1036 cumflex artery (LCX) was the most commonly intervened artery (n $=16 ; 43.2 \%)$ followed by left anterior descending $(\mathrm{n}=11 ; 29.7 \%)$ and right coronary artery $(\mathrm{n}=10 ; 27.1 \%)$. Mean diameter and mean length of stents were $3.3 \pm 0.9 \mathrm{~mm}$ and $18 \pm 6 \mathrm{~mm}$ respectively. The mean diameter and mean length of deflection balloon were 3 $\pm 0.5 \mathrm{~mm}$ and $20 \pm 5 \mathrm{~mm}$ respectively. Lesions were modified using aggressive pre-dilatation in all cases while $19.4 \%$ of lesion required cutting balloon for additional modification. Additional wire as buddy wire was used in $54 \%$ of cases while wire in main vessel acted as buddy wire in dedicated bifurcation stenting. Stent implantation was successful in 36 cases with success rate of $97.3 \%$; while in one case, stent could not be delivered using BDT. Stent was finally delivered using GuideZilla mother-in-child catheter. Overall failure rate was $2.7 \%$ which was contributed by extreme tortuosity, angulation, and severe calcification.

Conclusions: In selected cases of impassable lesions; the deflection balloon technique may provide a simple, convenient, and inexpensive solution without further need of additional hardwares except a buddy wire and a balloon.

Keywords: Balloon deflection technique; Bifurcation stenting; Chronic total occlusion; Balloon impassable situation

\section{Introduction}

Long and multiple lesions especially in the background of tortuous coronary artery often require multiple stents. Moreover, second stent might be needed to treat focal dissections at the distal edge of the deployed stent. The entry and passage of another stent through an already deployed stent becomes challenging because of poorly expanded stent struts, calcified tissue blocking passage of stent, underlying tortuosity, highly angulated takeoff of the vessel, and lack of coaxiality. Bulky stent transport systems and longer stent further adds to the problem. Mother-in-child catheter system (GuideLiner, GuideZilla), buddy wire, deeper intubation of guide catheter, and balloon anchor are few of the bailout solutions to such problems [1-3]. 


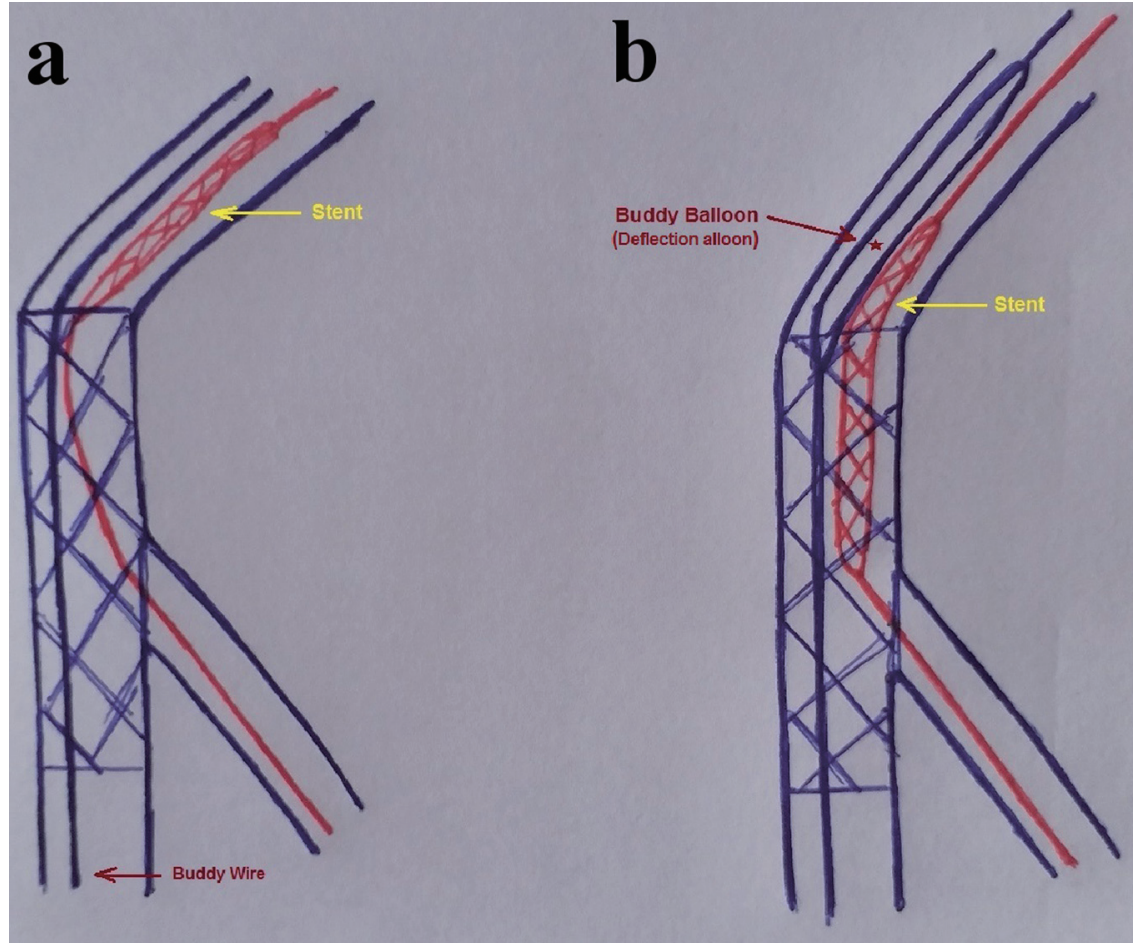

Figure 1. Schematic representation of deflection balloon technique. (a) Stent could not be crossed in side branch. (b) Deflection balloon at proximal edge of main vessel stent to facilitate side branch delivery.

\section{Materials and Methods}

\section{Design}

Between January 2014 to December 2019, 16,189 consecutive patients underwent percutaneous coronary intervention (PCI) at LPS Institute of Cardiology, GSVM Medical College, Kanpur, UP, India either transradial or transfemoral route among which balloon deflection technique (BDT) was used in 37 patients. Procedure was performed after obtaining written, informed consent from all patients. This study was approved by Institutional Review Board and was conducted in compliance with the ethical standards of the responsible institution on human subjects as well as with the Helsinki Declaration. Baseline demographics of patients including clinical (age, sex, clinical presentation), angiographic outcome (target lesion, type and character of lesion) and procedural data were recorded. Lesion was classified as type $\mathrm{A}, \mathrm{B} 1, \mathrm{~B} 2$ or $\mathrm{C}$ according to the American Heart Association/American College of Cardiology (AHA/ACC) criteria, which included length, calcification, chronicity, angulation, and tortuosity [4]. BDT was used when stent could not be delivered to target lesion (side branch in case of bifurcation, proximally or distally located lesion) due to various technical challenges such as: 1) origin of vessel having angulated takeoff; 2) proximal tortuosity of vessel; 3) calcification. In case of multiple indications, the key one leading to use of BDT was considered as primary. Safety endpoint was defined as absence of peri-procedural complications (perforation, dis- section, stent dislodgement). Intervention through transradial (TRI) or transfemoral (TFI) route was performed using standard protocol. Most of the procedures were performed through $6 \mathrm{~F}$ guiding catheter except dedicated bifurcation lesion and chronic total occlusion (CTO) where $7 \mathrm{~F}$ guide was used. Dual antiplatelet drugs (aspirin and either of ticagrelor, prasugrel, or clopidogrel) were administered orally before performing PCI and continued for at least 1 year following PCI. The choice of other drugs, intervention approach, equipment, and technique was left at operator's discretion.

\section{BDT}

One 0.014-inch Runthrough wire (Terumo Inc; Japan) was parked in distal part of target vessel as buddy wire beside the workhorse wire. On this buddy wire, the deflection balloon whose diameter was decided according to the diameter of proximal vessel in case of first stent, was parked at the site of resistance. The balloon was not inflated but kept in a way that it was partly inside the stent and partly outside. The buddy wire was firmly held while tracking the impassable stent over the workhorse wire. In case of provisional bifurcation stenting, the wire already parked in the main vessel acted as the buddy wire over which the deflection balloon (size of balloon was decided by size of deployed stent) was parked to facilitate the delivery of side branch stent. In cases of true bifurcation lesion, wire in side branch was used as buddy wire on which deflection balloon was parked to facilitate the delivery of main branch stent. The length of balloon was kept 


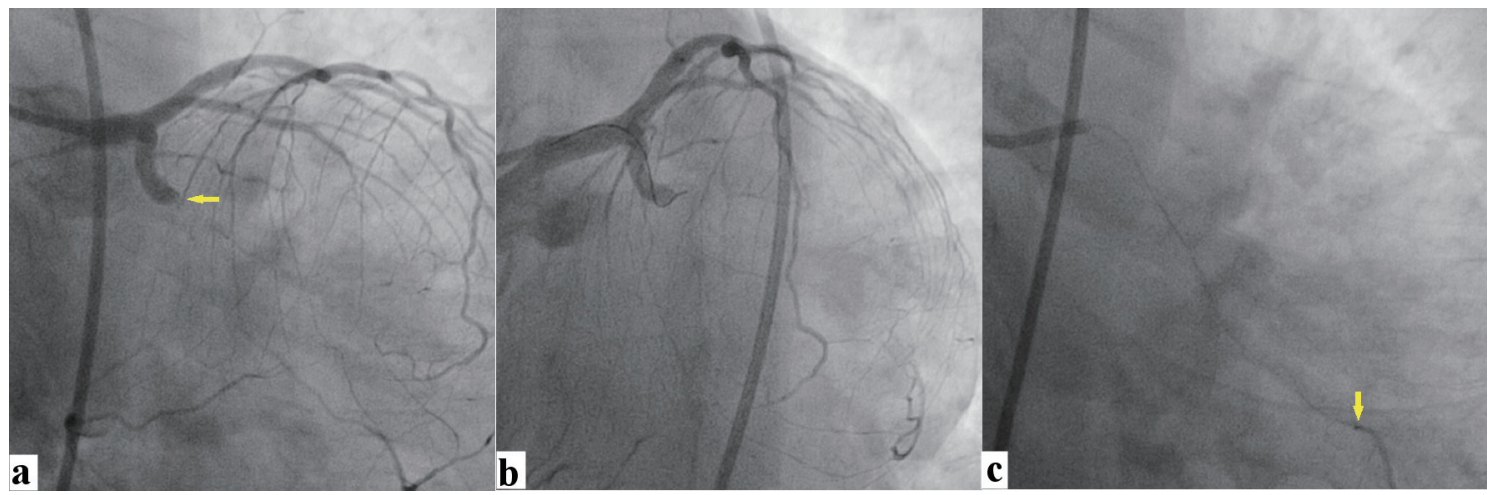

Figure 2. (a) Chronic total occlusion of left circumflex artery. (b) Wire was tried to cross using Finecross microcatheter (Asahi Inc., Japan). (c) After successfully crossing using Conquest Pro 12 (Asahi Inc, Japan), distal injection was given through Finecross which confirmed its true position (yellow arrow).
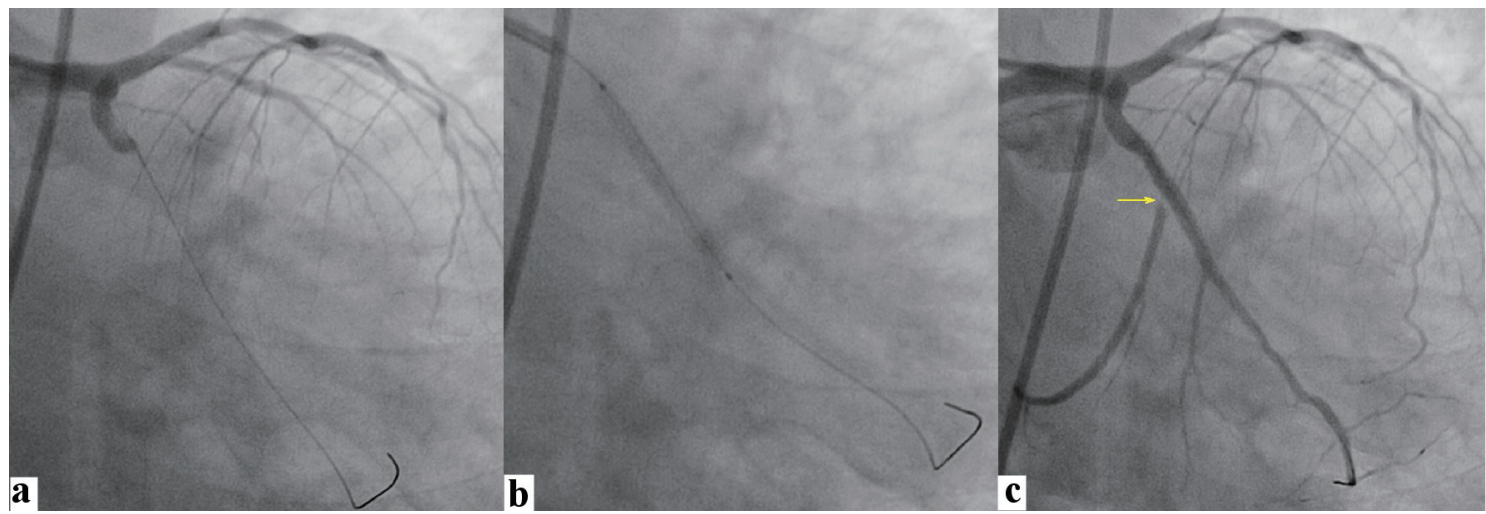

Figure 3. (a) Distal obtuse marginal branch was wired with Sion blue wire (Asahi Inc, Japan; A). (b) It was stented with $3.5 \times 38 \mathrm{En}$ deavour Resolute (Zotarolimus eluting stent, Medtronic; USA). (c) Ostial severe disease of distal circumflex branch (yellow arrow).

more than $12 \mathrm{~mm}$ to ease the delivery of stent. In dedicated and provisional stenting, the deflection balloon was chosen as non-compliant balloon as it was also used to perform final kissing inflation (FKI). It has been shown schematically in Figure 1, and one case of T stenting and protrusion (TAP) of chronically occluded left circumflex artery (LCX) has been shown in Figures 2 - 6.

\section{Statistical evaluation}

Statistical analyses were performed using SPSS 19.0 (SPSS

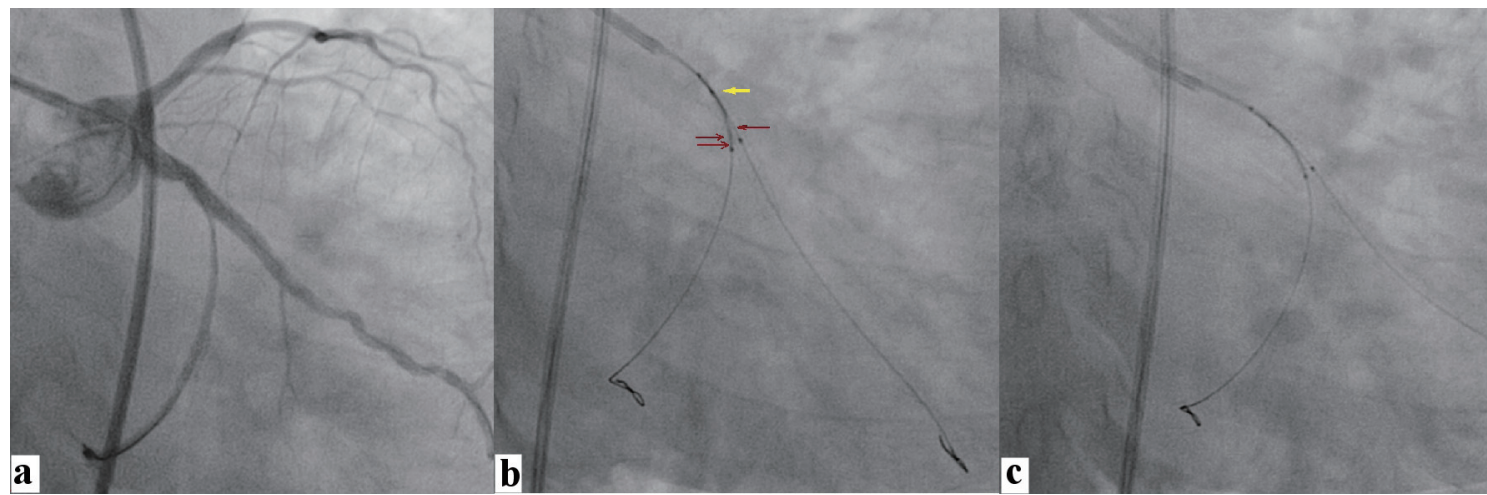

Figure 4. (a) Distal circumflex branch was rewired using Sion black wire. (b, c) After pre-dilatation of distal LCX, stent could not be negotiated into it because of tortuosity and calcification. A $3 \times 10$ Sprinter NC balloon (Medtronic Inc.; Japan) was used as deflection balloon which was kept little inside at proximal part of LCX (yellow arrow) and $2.75 \times 12 \mathrm{~mm}$ Endeavour Resolute stent was successfully delivered into distal LCX (double arrow). LCX: left circumflex artery. 


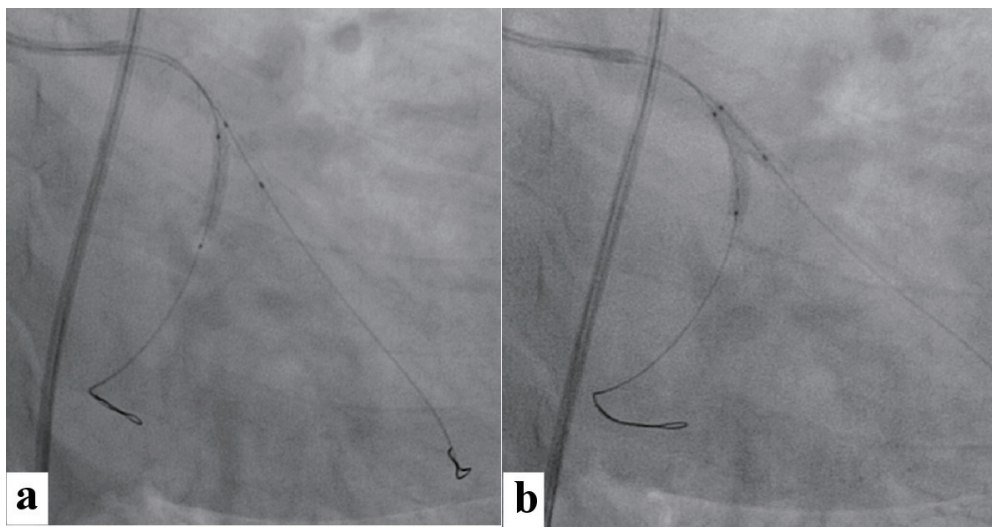

Figure 5. (a) Stent was positioned into distal LCX, deflection balloon was pushed and positioned across the distal LCX branch and stent was inflated. (b) Final kissing inflation was performed at 16 atm pressure after pulling LCX stent balloon little up. LCX: left circumflex artery.

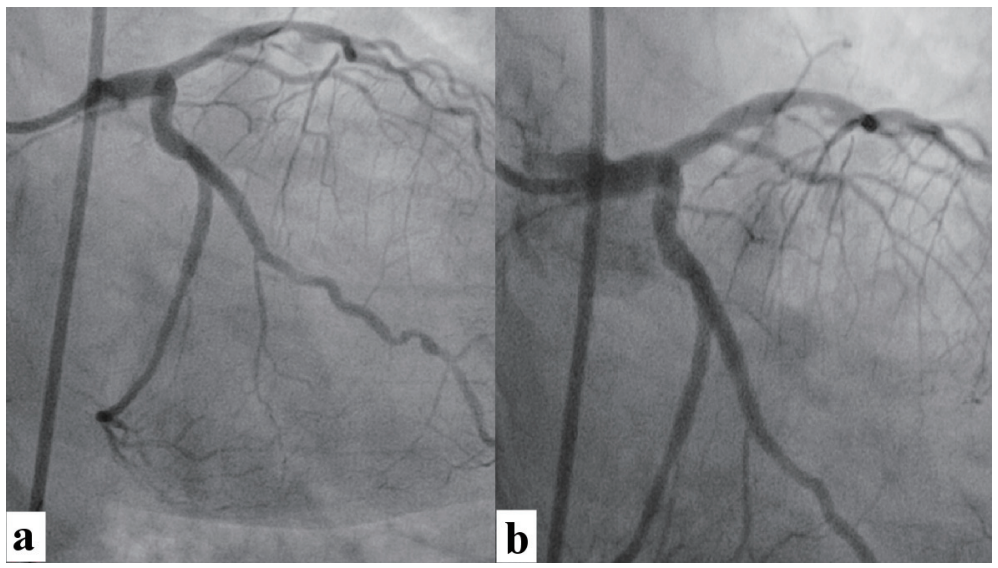

Figure 6. (a, b) Well apposed stent in main vessel and side branch with TIMI III flow. LCX: left circumflex artery.

Table 1. Baseline Characteristics of Patients $(N=37)$

\begin{tabular}{|ll}
\hline Variable & N $(\%)$ \\
\hline Age (years) & $75.4 \pm 6.5$ \\
Male/female & $28(75.7) / 9(24.3)$ \\
Risk factors for CAD & \\
$\quad$ Hypertension & $12(32.4)$ \\
$\quad$ Diabetes mellitus & $10(27.1)$ \\
$\quad$ Dyslipidemia & $6(16.2)$ \\
$\quad$ Smoking & $9(24.3)$ \\
Indication for PCI & \\
\multicolumn{1}{c}{ STEMI } & $5(13.5)$ \\
NSTEMI & $9(24.4)$ \\
UA & $4(10.8)$ \\
CCS & $19(51.3)$ \\
\hline
\end{tabular}

CAD: coronary artery disease; PCI: percutaneous coronary intervention; STEMI: ST-segment elevation myocardial infarction; NSTEMI: non-ST-segment elevation myocardial infarction; UA: unstable angina; CCS: chronic coronary syndrome.
Inc., Chicago, IL, USA). All continuous variables were expressed as the mean \pm standard deviation, and categorical data were recorded as percentage.

\section{Results}

Baseline data of the patients are presented in Table 1. The mean age was $75.4 \pm 6.5$ years. Hypertension was commonest risk factor $(\mathrm{n}=12 ; 32.4 \%)$, followed by diabetes mellitus $(\mathrm{n}=10 ; 27.1 \%)$, smoking $(\mathrm{n}=9 ; 24.3 \%)$, and dyslipidemia $(\mathrm{n}=6 ; 16.2 \%)$. The commonest clinical presentation was chronic coronary syndrome (CCS) $(\mathrm{n}=19 ; 51.3 \%)$ followed by non-ST-segment elevation myocardial infarction (NSTEMI) $(\mathrm{n}=9 ; 24.4 \%)$, ST-segment elevation myocardial infarction (STEMI) $(\mathrm{n}=5 ; 13.5 \%)$, and UA $(\mathrm{n}=10$; $10.8 \%$ ). Type B2 lesion was commonest (45.9\%) followed by type C (35.2\%) and B1 (18.9\%). The commonest indication for BDT was angulation (27.1\%) followed by tortuosity $(24.3 \%)$, chronic total occlusion $(17.9 \%)$, calcification $(18.9 \%)$, and distally located lesion $(8.1 \%$, Table 2$)$. The LCX was the most commonly intervened artery in $43.2 \%$ 
Table 2. Angiographic Characteristics and Procedural Outcome of Patients Who Underwent PCI Using Balloon Deflection Technique $(\mathrm{N}=37)$

\begin{tabular}{|c|c|}
\hline Variable & $\mathbf{N}(\%)$ \\
\hline Success/failure & $36(97.3) / 1(2.7)$ \\
\hline \multicolumn{2}{|l|}{ Target vessel } \\
\hline LAD & $11(29.7)$ \\
\hline LCX & $16(43.2)$ \\
\hline RCA & $10(27.1)$ \\
\hline \multicolumn{2}{|l|}{ Lesion characteristics } \\
\hline Calcification & $7(18.9)$ \\
\hline Tortuosity & $9(24.3)$ \\
\hline Angulation & $10(27.1)$ \\
\hline CTO & $8(21.6)$ \\
\hline Distally located lesion & $3(8.1)$ \\
\hline \multicolumn{2}{|l|}{ Type of lesion } \\
\hline $\mathrm{B} 1$ & $7(18.9)$ \\
\hline B2 & $17(45.9)$ \\
\hline $\mathrm{C}$ & $13(35.2)$ \\
\hline Mean diameter of the stents & $3.3 \pm 0.9 \mathrm{~mm}$ \\
\hline Mean length of stents & $18 \pm 6 \mathrm{~mm}$ \\
\hline Mean diameter of defection balloon & $3 \pm 0.5 \mathrm{~mm}$ \\
\hline Mean length of defection balloon & $20 \pm 0.5 \mathrm{~mm}$ \\
\hline \multicolumn{2}{|l|}{ Lesion preparation } \\
\hline Aggressive pre-dilatation & $36(100)$ \\
\hline Cutting balloon modification of the lesion & $7(19.4)$ \\
\hline Buddy wire technique & $20(54.1)$ \\
\hline \multicolumn{2}{|l|}{ Complications } \\
\hline Peri-procedural (dissection, stent dislodgement) & $1(3.7)$ \\
\hline In hospital (MI, ST) & $0(0.0)$ \\
\hline Mortality at 30 day & $0(0.0)$ \\
\hline
\end{tabular}

LAD: left anterior descending artery; LCX: left circumflex coronary artery; RCA: right coronary artery; CTO: chronic total occlusion; PCI: percutaneous coronary intervention; MI: myocardial infarction; ST: stent thrombosis.

cases followed by left anterior descending (LAD) and right coronary artery (RCA) in $29.7 \%$ and $27.1 \%$ cases, respectively (Table 2). Mean diameter and mean length of stents were $3.3 \pm 0.9 \mathrm{~mm}$ and $18 \pm 6 \mathrm{~mm}$ respectively. The mean diameter and mean length of deflection balloon were $3 \pm$ $0.5 \mathrm{~mm}$ and $20 \pm 5 \mathrm{~mm}$ respectively. Lesions were modified using aggressive pre-dilatation in all cases while $19.4 \%$ of lesion required cutting balloon for additional modification. Buddy wire was used in 54\% of cases as wire in main vessel acted as buddy wire in dedicated bifurcation stenting. Stent implantation was successful in 36 out of 37 patients with success rate of $97.3 \%$; while in one case, stent could not be delivered using BDT. Stent was finally delivered using GuideZilla mother-in-child catheter. Overall failure rate was $2.7 \%$ which were contributed by extreme tortuosity, angulation, and severe calcification (Table 2).

\section{Discussion}

Calcified lesions are one of the challenging substrate as they can be very difficult to treat as tracking the balloon and stent catheters through the lesions can be very difficult. Calcification in the background of tortuosity further adds to problem [5]. Lesion modification by debunking using rotablation or laser are few of the options but are technically demanding [4]. They may be relatively contraindicated in the background of tortuosity as wire bias may be risk for vessel perforation. In selected cases, cutting balloons are useful but their profile and rigid blades may hamper its trackability in tightly calcified lesions [6, 7]. Buddy wire using two or more wires along with more supportive guiding catheters like Amplatz may provide better trackability and deliverability.

Sometime, second 0.014-inch guidewire may be parked 
distally which acts as buddy wire to deliver the stent. It provides a smooth rail for a stent to advance along the luminal wall without snagging; but at times they may not garner enough support especially in case of proximal tortuosity/angulations, distally located lesions in the vessel, stenting of a lesion distally located from a previously implanted stent and from a coronary segment with both calcification and sharp bend [8]. Inflation of deflection balloon over the buddy wire provided extra support as vessel was little straightened which facilitated the delivery of stent at the target site.

The problem encountered in our patients was geometric bias of the wires caused by calcified eccentric plaque and tortuosity which impaired the trackability of stents. Simple buddy wire technique failed in our patients which were circumvented by using BDT. Distal buddy balloon was also used initially in few of the cases but they were not successful in few cases which were also sorted out using BDT. The buddy balloon occupied the eccentric dead-end space just proximal to the lesion, and was strong enough to direct the second balloon/stent over to the lesion surface smoothly. Therefore, out default approach in such situation was deflection technique with good success (97.3\%). Plaque modification with rotational atherectomy should have solved this problem easily. However, it adds additional costs and procedure time as well as its associated potential complications. In case of failure, the stent was delivered using GuideZilla mother-in-child catheter system. This technique was also reported by Abernethy et al with excellent success though only in five cases, but this was only technique which provided them breakthrough [9]. Another advantage of BDT was that the same deflection balloon can also be used for final kissing inflation without adding further cost, thereby making it cost effective approach as well. Furthermore, it can be performed well through $6 \mathrm{~F}$ guide catheter as it can easily accommodate either two balloons or one stent and one balloon. Such technique can also be applied in other occasions such as difficulty in passing a balloon catheter into a stent for post deployment high-pressure dilatation and difficulty in passing a retrieval catheter or aspiration catheter through a stent at the end of the procedure after using distal protection devices.

However, there are certain limitations to this technique such as manipulation of multiple wires and balloon catheters which may also cause endothelial injury, and rupture of new plaque causing thrombosis or acute occlusion. One should be cautious enough to pull the deflection balloon and wire before stent deployment to prevent their jailing in case of distally located lesion and dedicated bifurcation stenting. Other options should be to secure more stable guide, larger guide or one with different curve to achieve better backup support, and motherin-child catheter. Mother-in-child system is very costly and also associated with potential complications [3]. The choice of approach to impassable lesions depends on the underlying cause, availability of other hardwares, and operator's experience. In selected cases, the deflection balloon technique may provide a simple, convenient, and inexpensive solution.

\section{Conclusions}

In selected cases of impassable lesions, the deflection balloon technique may provide a simple, convenient, and inexpensive solution without further need of additional hardwares except a buddy wire and a balloon.

\section{Acknowledgments}

None to declare.

\section{Financial Disclosure}

None to declare.

\section{Conflict of Interest}

None to declare.

\section{Informed Consent}

Informed consent was obtained.

\section{Author Contributions}

Substantial contributions to the conception or design of the work; or the acquisition, analysis, or interpretation of data for the work: PK, SKS, MR, UP, AKS, RT, PS and VK. Drafting the work or revising it critically for important intellectual content: PK, SKS, NUS, MR, UP, AKS, RT and VK. Agreement to be accountable for all aspects of the work in ensuring that questions related to the accuracy or integrity of any part of the work are appropriately investigated and resolved: PK, MR, PS, UP, RT and VK. Final approval of the version to be published: NUS, SKS, MK, PS, UP, AKS, RT and VK.

\section{Data Availability}

The authors declare that data supporting the findings of this study are available within the article.

\section{References}

1. Di Mario C, Ramasami N. Techniques to enhance guide catheter support. Catheter Cardiovasc Interv. 2008;72(4):505512.

2. Saeed B, Banerjee S, Brilakis ES. Percutaneous coronary intervention in tortuous coronary arteries: associated complications and strategies to improve success. J Interv Cardiol. 2008;21(6):504-511.

3. Chan PH, Alegria-Barrero E, Foin N, Paulo M, Lindsay AC, Viceconte N, Di Mario C. Extended use of the GuideLiner in complex coronary interventions. EuroIntervention. 2015;11(3):325-335.

4. Satler LF. The frustrations of coronary stenting. Cathet 
Cardiovasc Diagn. 1995;35(3):216-217.

5. Buchwald AB, Werner GS, Unterberg C, Voth E, Kreuzer $\mathrm{H}$, Wiegand V. Restenosis after excimer laser angioplasty of coronary stenoses and chronic total occlusions. Am Heart J. 1992;123(4 Pt 1):878-885.

6. Orford JL, Fasseas P, Denktas AE, Hammes L, Garratt KN, Berger PB, Holmes DR, et al. Safety and efficacy of cutting balloon angioplasty: the Mayo Clinic experience. J Invasive Cardiol. 2002;14(12):720-724.

7. Lee MS, Singh V, Nero TJ, Wilentz JR. Cutting balloon angioplasty. J Invasive Cardiol. 2002;14(9):552-556.

8. Burzotta F, Trani C, Mazzari MA, Mongiardo R, Rebuzzi AG, Buffon A, Niccoli G, et al. Use of a second buddy wire during percutaneous coronary interventions: a simple solution for some challenging situations. J Invasive Cardiol. 2005;17(3):171-174.

9. Abernethy WB, 3rd, Choo JK, Oesterle SN, Jang IK. Balloon deflection technique: A method to facilitate entry of a balloon catheter into a deployed stent. Catheter Cardiovasc Interv. 2000;51(3):312-313. 